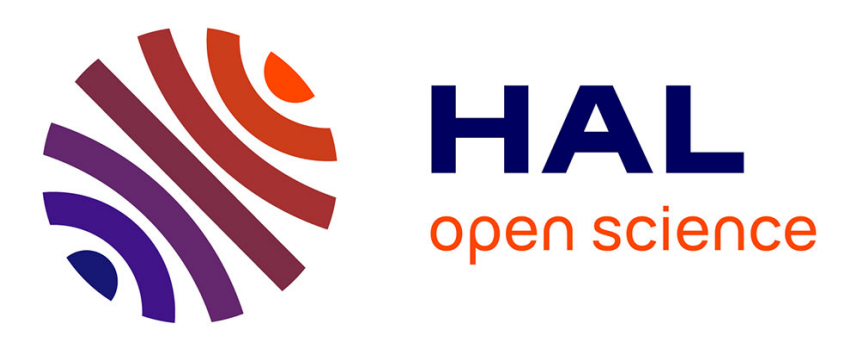

\title{
Optimizing Prediction Dynamics With Saturated Inputs for Robust Model Predictive Control
}

\author{
Hoaï-Nam Nguyen
}

\section{To cite this version:}

Hoaï-Nam Nguyen. Optimizing Prediction Dynamics With Saturated Inputs for Robust Model Predictive Control. IEEE Transactions on Automatic Control, 2021, 66 (1), pp.383-390. 10.1109/TAC.2020.2979399 . hal-03129828

\section{HAL Id: hal-03129828 \\ https://hal-ifp.archives-ouvertes.fr/hal-03129828}

Submitted on 3 Feb 2021

HAL is a multi-disciplinary open access archive for the deposit and dissemination of scientific research documents, whether they are published or not. The documents may come from teaching and research institutions in France or abroad, or from public or private research centers.
L'archive ouverte pluridisciplinaire HAL, est destinée au dépôt et à la diffusion de documents scientifiques de niveau recherche, publiés ou non, émanant des établissements d'enseignement et de recherche français ou étrangers, des laboratoires publics ou privés. 


\title{
Optimizing prediction dynamics with saturated inputs for robust model predictive control
}

\author{
H.-N. Nguyen ${ }^{\dagger}$
}

\begin{abstract}
A model predictive control algorithm based on offline optimization of prediction dynamics enables an efficient online computation. However, the price for this efficiency is a reduction in the degree of optimality. This paper presents a new method for overcoming this weakness, yielding a significant improvement in the degree of optimality, and achieving this with no increase in online computational load. Two numerical examples with comparison to earlier solutions from the literature illustrate the effectiveness of the proposed algorithm.
\end{abstract}

\section{INTRODUCTION}

Model predictive control (MPC) is one strategy that can handle input/state constraints while providing near optimal performance and guaranteeing stability. The basic idea of MPC can be summarized as follows: at each sampling interval, an explicit process model is used to predict the future plant behavior, and a constrained optimization problem is then solved to obtain a sequence of future manipulated variable control adjustments. Only the first input in the optimal sequence is then applied to the plant, and the entire calculation is repeated for the next sampling interval [1], [2]. In spite of the increased computational power of control computers, MPC is at present mainly suitable for low-order, nominally linear systems. It is well known [3], [1] that solving the MPC optimization problem incorporating robustness to model uncertainty can be very computationally demanding, as it is NP hard. In particular in the robust min-max MPC approach [3], where a sequence of control actions is obtained which ensures the constraint satisfaction along the predicted trajectory of the plant for any possible uncertainty, and minimizes the worst case performance index of the predicted evolution of the plant. However, the computational

$\dagger$ Control, Signal and Systems Department - IFP Energies Nouvelles, Rond-point de l'echangeur de Solaize BP3, 69360 Solaize, France hoai-nam.nguyendifpen.fr 
load remains prohibitive for fast sampling applications. In [4], it was shown that the robust min-max MPC with a linear cost is equivalent to a multiparametric linear program. The control is a piecewise affine function of the state over a polyhedral partition of the state space, and the computational effort of the min-max MPC is moved offline. However, the prohibitive online storage and search requirements of this approach limit its application to low order systems and/or short prediction horizon.

In the last decades, several works aiming at reducing the online computational complexity have been reported. For example, in [2] the online optimization problem is formulated as a semidefinite program (SDP). At each time instant, a linear state feedback law is obtained. In [5], an offline ellipsoidal min-max MPC scheme was proposed by using a sequence of linear state feedback laws that correspond to a sequence of nested invariant ellipsoids. In [6], a robust triple mode MPC algorithm was considered by introducing an additional mode in dual mode MPC with a large feasible region and good performance. The main challenge is to find a suitable linear time varying control law which enlarges feasibility without too much detriment to performance.

In [7], [8], a significant reduction in online computational load is achieved. The basic idea is to use an autonomous augmented system to generate predictions, the state of which consists of a vector of degree of freedom in predictions appended to the plant state. The augmented state is required to lie in an augmented ellipsoid, which is invariant under the prediction dynamics and constraint-admissible with respect to constraints. The invariant ellipsoid is computed offline. The online minimization of the predicted performance index can be solved efficiently by using a univariate Newton-Raphson iteration. In [9], [10], the approach was improved by allowing the parameters of the dynamic feedback laws to be variables in the offline optimization. As a result the size of the feasible set can be significantly enlarged. However, the formulation in [9], [10] is non-convex, hence leads to no guarantee on convergence of the solution. In [11], the approach is further optimized and formulated into a convex problem. A weakness of the control law [11] is that the full control range is rarely exploited. Therefore the time to regulate the plant to the origin is much longer than necessary. For nominal systems, some attempts to overcome this drawback can be found in [7].

Inspired by advances in control methods with actuator saturation [12], the paper aims to improve the performance of the prediction dynamics approach for robust MPC. The main contributions are that: (i) this is the first time, to the best of the author's knowledge, a saturated nonlinear feedback policy is used in the MPC context; (ii) by allowing the parameters of the 
dynamic feedback laws to depend not only on the system uncertainty, but also on the saturated inputs, the control range is fully exploited. It is shown that the feasible set can be equal to the maximal invariant ellipsoid under any saturated feedback law. Despite a significant improvement in the performance, the proposed approach does not induce any extra online computational load.

This paper is organized as follows. Section II covers the problem formulation and preliminaries. Section III is dedicated to the prediction dynamics with saturated inputs. Then in Section IV results on the design of the new stabilizing control law are presented. Two simulated examples with comparison to earlier solutions are evaluated in Section $\mathrm{V}$ before drawing the conclusions in Section VI.

Notation: A positive-definite (semi-definite) matrix $P$ is denoted by $P \succ 0(P \succeq 0)$. $\mathbf{0}_{n \times m}$ is the zero matrix of dimension $n \times m$, and $\mathbf{I}_{n}$ is the identity matrix of dimension of $n \times n$. For a given $P \succeq 0, \mathcal{E}(P)$ represents the following ellipsoid $\mathcal{E}(P)=\left\{x \in \mathbb{R}^{n}: x^{T} P x \leq 1\right\}$. For symmetric matrices, the symbol $(*)$ denotes each of its symmetric block. The expression $\sum_{l=1}^{r} \eta_{l} v_{l}$ with a given set of vectors $v_{l}, l=\overline{1, r}$, and $\sum_{l=1}^{r} \eta_{l}=1, \eta_{l} \geq 0$ is called the convex hull of $v_{l}, l=\overline{1, r}$, and is denoted as $C o\left\{v_{l}, l=\overline{1, r}\right\}$.

\section{Problem Statement And Preliminaries}

\section{A. Problem Statement}

Consider the following uncertain and/or time-varying system

$$
x(k+1)=A(k) x(k)+B(k) u(k)
$$

with constraints on the input $u \in \mathbb{R}^{m}$, and on the measured state $x \in \mathbb{R}^{n}$

$$
\begin{aligned}
& -1 \leq u_{j} \leq 1, \forall j=1,2, \ldots, m \\
& x \in \mathcal{L}(F), \mathcal{L}(F)=\left\{x \in \mathbb{R}^{n}:\left|f_{l} x\right| \leq 1, \forall l=\overline{1, n_{c}}\right\}
\end{aligned}
$$

where $f_{l}$ is the $l$ th row of the matrix $F \in \mathbb{R}^{n_{c} \times n}$. For simplicity, element-wise inequalities (2), (3) are considered. However, the technique in the paper can be extended to more general mixed state/input constraints.

The matrices $A(k), B(k)$ are linearly parameterized, i.e.,

$$
A(k)=\sum_{i=1}^{q} \theta_{i}(k) A_{i}, \quad B(k)=\sum_{i=1}^{q} \theta_{i}(k) B_{i}
$$


where $A_{i} \in \mathbb{R}^{n \times n}, B_{i} \in \mathbb{R}^{n \times m}$ are known matrices. $\theta(k)=\left[\theta_{1}(k) \theta_{2}(k) \ldots \theta_{q}(k)\right]$ is a vector of parametric uncertainties, that satisfies

$$
\theta(k) \in \Theta:\left\{\sum_{i=1}^{q} \theta_{i}=1, \theta_{i} \geq 0, \forall i=\overline{1, q}\right\}
$$

It is underlined that $\theta(k)$ is unknown and time-varying.

Denote $x(k+t \mid k)$ and $u(k+t \mid k), t=0,1,2, \ldots$, respectively, as the predicted states and the predicted control inputs from time $k$. The robust control design problem is to select input $u(k+t \mid k), t=0,1, \ldots$, that steers the state to the origin, while satisfying the constraints (3), and solving the following minimax problem

$$
\begin{aligned}
& \min _{\{u(k \mid k), u(k+1 \mid k), \ldots,\}} \max _{\theta(k) \in \Theta}\{J(k)\}, \\
& J(k)=\sum_{t=0}^{\infty}\left(x(k+t \mid k)^{T} Q x(k+t \mid k)+u(k+t \mid k)^{T} R u(k+t \mid k)\right)
\end{aligned}
$$

where $Q \succeq 0, R \succ 0$ are the state and input weighting matrices, respectively.

Remark 1: Without (2), (3), it is well known [13] that (6) is a robust linear quadratic (LQ) regulator problem, for which the solution is the linear state feedback controller

$$
u(k+t \mid k)=K x(k+t \mid k), t=0,1, \ldots
$$

where $K \in \mathbb{R}^{m \times n}$ is found by solving a SDP problem. In the presence of (2), (3), the problem (6) is intractable due to the need of guaranteeing (2), (3) for the infinite number of constraints.

\section{B. Previous Works: Prediction Dynamics with Linear Feedback}

To guarantee feasibility, the constraints (2), (3) must be satisfied along predicted trajectories of the plant, i.e., $x(k+t \mid k) \in \mathcal{L}(F)$ and $u(k+t \mid k) \in[-1,1], \forall t=0,1, \ldots$ These constraints impose a heavy computational cost. A way to reduce it is to express the degrees of freedom in predicted inputs as a perturbation sequence $c(k+t \mid k) \in \mathbb{R}^{m}$ on the control law (8)

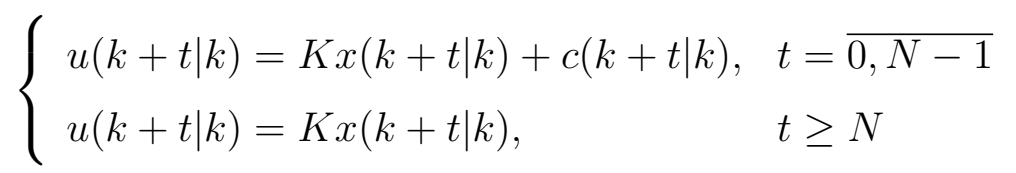

where $N$ is the control horizon. Beyond $N$, we can set $c(k+t \mid k)=0$ assuming the robust LQ control (8) is feasible onwards [8]. Using (9), the corresponding predicted trajectories are 
generated by an autonomous system, the state of which is the predicted state of the plant augmented by the perturbation sequence

$$
\left[\begin{array}{c}
x(k+t+1 \mid k) \\
\zeta(k+t+1 \mid k)
\end{array}\right]=\Omega(k+t \mid k)\left[\begin{array}{l}
x(k+t \mid k) \\
\zeta(k+t \mid k)
\end{array}\right]
$$

with $\zeta(k \mid k)=\left[c(k \mid k)^{T} c(k+1 \mid k)^{T} \ldots c(k+N-1 \mid k)^{T}\right]^{T}$

$$
\Omega(k)=\left[\begin{array}{cc}
A(k)+B(k) K & B(k) C_{c} \\
\mathbf{0}_{m n_{c} \times n} & A_{c}
\end{array}\right], A_{c}=\left[\begin{array}{ccccc}
\mathbf{0}_{m} & \mathbf{I}_{m} & \mathbf{0}_{m} & \ldots & \mathbf{0}_{m} \\
\mathbf{0}_{m} & \mathbf{0}_{m} & \mathbf{I}_{m} & \ldots & \mathbf{0}_{m} \\
\vdots & \vdots & \vdots & \ddots & \vdots \\
\mathbf{0}_{m} & \mathbf{0}_{m} & \mathbf{0}_{m} & \ldots & \mathbf{I}_{m} \\
\mathbf{0}_{m} & \mathbf{0}_{m} & \mathbf{0}_{m} & \ldots & \mathbf{0}_{m}
\end{array}\right], C_{c}=\left[\begin{array}{c}
\mathbf{I}_{m} \\
\mathbf{0}_{m} \\
\vdots \\
\mathbf{0}_{m}
\end{array}\right]^{T}
$$

In [9], [10], the control strategy (9), (10) is improved by considering $A_{c}, C_{c}$ as parameters to be optimized offline. As a result, the size of the domain of attraction can be considerably enlarged. However, the formulation in [9], [10] leads to a nonconvex optimization problem. The method is further optimized in [11] by: (i) Replacing a constant $A_{c}$ with a time-varying $A_{c}(k)$. Hence the predicted perturbation sequence is allowed to depend on future model uncertainties; (ii) Reformulating the offline optimization as a convex SDP problem. Therefore the problems of computational complexity, and of convergence caused by non-convex constraints are eliminated.

With these changes the predicted inputs are generated by the dynamic state feedback law

$$
u(k+t \mid k)=K x(k+t \mid k)+C_{c} c(k+t \mid k), t=0,1, \ldots
$$

with $c(k+t+1 \mid k)=A_{c}(k+t \mid k) c(k+t \mid k)$. Using the control law (11), two notable results are shown: (i) There is no advantage to be gained by using $N>n$ in terms of the size of the domain of attraction; (ii) The maximal invariant ellipsoidal approximation of the domain of attraction for the plant state under the dynamic feedback control law (11) can be equal to the maximal invariant ellipsoidal set under any linear state feedback law.

A significant reduction in online computational load can be achieved by computing an invariant ellipsoidal approximation of the domain of attraction for the augmented state $\left[x(k \mid k)^{T} \zeta(k \mid k)^{T}\right]$ offline [11]. If $\left[x(k \mid k)^{T} \zeta(k \mid k)^{T}\right]$ is imposed to lie with this invariant ellipsoid, then the entire predicted trajectory will be feasible. This constitutes a quadratic constraint on $\zeta$, which, combined with the cost function (7), leads to an efficient online optimization, since it requires only the computation of the unique negative root of a well behaved polynomial [8]. The price for this 
efficiency is a reduction in the degree of optimality, since the full control range is rarely exploited. The main objective of this paper is to remove this drawback, yielding a significant improvement in the performance, and without increasing in online computational cost. For this purpose, some preliminary results are recalled in the next section.

\section{Preliminaries}

Definition 1: A set $\mathcal{E}(P)$ is said to be robustly invariant for system (1) if for any $x(k) \in \mathcal{E}(P)$, $\forall k \geq 0$, there exists $u(k)=f(x(k))$ such that $x(k+1) \in \mathcal{E}(P), \forall \theta(k)$. Furthermore, if $\mathcal{E}(P) \subseteq \mathcal{L}(F)$ and $-1 \leq u(k) \leq 1$, then $\mathcal{E}(P)$ is robustly invariant and constraint-admissible.

Lemma 1: [14] For a vector $f_{0} \in \mathbb{R}^{1 \times n}$ and a matrix $P \succeq 0, \mathcal{E}(P) \subseteq \mathcal{L}\left(f_{0}\right)$ if and only if $f_{0} P^{-1} f_{0}^{T} \leq 1$.

The function $\operatorname{sat}(u): \mathbb{R}^{m} \rightarrow \mathbb{R}^{m}$ is defined as

$$
\begin{aligned}
& \operatorname{sat}(u)=\left[\operatorname{sat}\left(u_{1}\right) \operatorname{sat}\left(u_{2}\right) \ldots \operatorname{sat}\left(u_{m}\right)\right]^{T}, \\
& \operatorname{sat}\left(u_{j}\right)= \begin{cases}-1, & \text { if } u_{j} \leq-1 \\
u_{j}, & \text { if }-1 \leq u_{j} \leq 1, \forall j=\overline{1, m} \\
1, & \text { if } u_{j} \geq 1\end{cases}
\end{aligned}
$$

Define $\mathcal{M}=\{1,2, \ldots, m\}$, and $\mathcal{V}$ as the set of all subsets of $\mathcal{M}$, i.e., $\mathcal{V}=\{S: S \subseteq \mathcal{M}\}$. Note that the empty set belongs to $\mathcal{V}$. Define also $S^{c}$ as the complementary of $S$ in $\mathcal{M}$, i.e., $S^{c}=\{i \in \mathcal{M}: i \notin S\}$. For example, if $m=2$, then $\mathcal{M}=\{1,2\}$ and $\mathcal{V}=\left\{S_{1}, S_{2}, S_{3}, S_{4}\right\}$ with

$$
\begin{aligned}
& S_{1}=\emptyset, S_{2}=\{1\}, S_{3}=\{2\}, S_{4}=\{1,2\}, \\
& S_{1}^{c}=\{1,2\}, S_{2}^{c}=\{2\}, S_{3}^{c}=\{1\}, S_{4}^{c}=\emptyset
\end{aligned}
$$

Denote $e_{j}$ as the $j$ th standard basis of $\mathbb{R}^{m}$, i.e.,

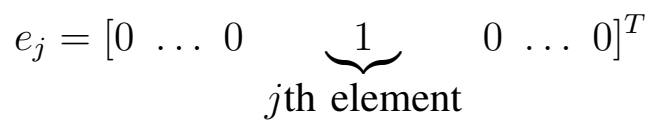

Associated to $S_{s}, \forall s=\overline{1,2^{m}}$, consider the following scalars $v_{s(j)}, \forall j=\overline{1, m}$,

$$
\begin{cases}-1 \leq v_{s(j)} \leq 1, & \text { if } j \in S_{s} \\ v_{s(j)}=0, & \text { if } j \notin S_{s}\end{cases}
$$

The following lemma is taken from [12]. It has been proposed originally in [15], [14].

Lemma 2: [12] With $v_{s(j)}$ defined as in (14), the following equation holds

$$
\operatorname{sat}(u) \in C_{o}\left\{\sum_{j \in S_{s}^{c}} e_{j} u_{j}+\sum_{j \in S_{s}} e_{j} v_{s(j)}, s=\overline{1,2^{m}}\right\}
$$


For example, if $m=2$, we have

$$
\left[\begin{array}{l}
\operatorname{sat}\left(u_{1}\right) \\
\operatorname{sat}\left(u_{2}\right)
\end{array}\right] \in \operatorname{Co}\left\{\left[\begin{array}{l}
u_{1} \\
u_{2}
\end{array}\right],\left[\begin{array}{c}
v_{2(1)} \\
u_{2}
\end{array}\right],\left[\begin{array}{c}
u_{1} \\
v_{3(2)}
\end{array}\right],\left[\begin{array}{l}
v_{4(1)} \\
v_{4(2)}
\end{array}\right]\right\}
$$

Denote $K_{(j)}$ the $j$ th row of $K$ in (8), and $\mathcal{B}_{i(j)}$ as the $j$ th column of $\mathcal{B}_{i}, \forall i=\overline{1, q}, \forall j=\overline{1, m}$. The following corollary is a direct consequence of Lemma 1, and Lemma 2.

Corollary 1: Suppose that a symmetric matrix $Q \in \mathbb{R}^{n \times n}$, matrices $W_{s} \in \mathbb{R}^{m \times n}$ satisfy the following linear matrix inequalities (LMI), $\forall i=\overline{1, q}, \forall s=\overline{1,2^{m}}$

$$
\begin{aligned}
& {\left[\begin{array}{c}
Q \\
(*) \\
\left(A_{i}+\sum_{j \in S_{s}^{c}} B_{i(j)} K_{(j)}\right) Q+\sum_{j \in S_{s}} B_{i(j)} W_{s(j)} \\
Q
\end{array}\right] \succeq 0} \\
& {\left[\begin{array}{cc}
1 & W_{s(j)} \\
W_{s(j)}^{T} & Q
\end{array}\right] \succeq 0, \forall j=\overline{1, m}} \\
& {\left[\begin{array}{cc}
1 & f_{l} Q \\
Q f_{l}^{T} & Q
\end{array}\right] \succeq 0, \forall l=\overline{1, n_{c}}}
\end{aligned}
$$

where $W_{s(j)}$ is the $j$ th row of $W_{s}$. Then $\mathcal{E}\left(Q^{-1}\right)$ is robustly invariant for $x(k+1)=A(k) x(k)+$ $B(k) \operatorname{sat}(K x(k))$ and constraint-admissible with respect to (2), (3).

Proof: Define $L_{s(j)}=W_{s(j)} Q^{-1}$. Using (17), one gets, $\forall s=\overline{1,2^{m}}$

$$
\left[\begin{array}{cc}
1 & L_{s(j)} Q \\
Q L_{s(j)}^{T} & Q
\end{array}\right] \succeq 0, \forall j=\overline{1, m}
$$

Or equivalently, $L_{s(j)} Q L_{s(j)}^{T} \leq 1$. Using Lemma 1, it follows that $\mathcal{E}\left(Q^{-1}\right) \subseteq \mathcal{L}\left(L_{s(j)}\right)$.

Note that (18) implies $\mathcal{E}\left(Q^{-1}\right) \subseteq \mathcal{L}(F)$. Hence $\mathcal{E}\left(Q^{-1}\right)$ is constraint-admissible with respect to (2), (3). It remains to prove that $\mathcal{E}\left(Q^{-1}\right)$ is robustly invariant. Using Schur complement, rewrite (16) as $Q-Q \mathcal{J}_{i s}^{T} Q^{-1} \mathcal{J}_{i s} Q \succeq 0$, or equivalently

$$
x^{T}(k) Q^{-1} x(k)-x^{T}(k) \mathcal{J}_{i s}^{T} Q^{-1} \mathcal{J}_{i s} x(k) \geq 0
$$

with $\mathcal{J}_{i s}=A_{i}+\sum_{j \in S_{s}^{c}} B_{i(j)} K_{(j)}+\sum_{j \in S_{s}} B_{i(j)} L_{s(j)}$. Using Lemma 2, one gets, $\forall i=\overline{1, q}$

$$
A_{i} x(k)+B_{i} \operatorname{sat}(K x(k)) \in C o\left\{\mathcal{J}_{i s} x(k), \forall s=\overline{1,2^{m}}\right\}
$$

It follows that $x^{T}(k) Q^{-1} x(k)-x(k+1) Q^{-1} x(k+1) \leq 0$. Hence $\mathcal{E}\left(Q^{-1}\right)$ is robustly invariant. 
In the interest of the size of the domain of attraction, which is proportional to logdet $(Q)$, the set $\mathcal{E}\left(Q^{-1}\right)$ should be maximized. This can be done by solving the following SDP problem

$$
\begin{aligned}
& \max _{Q, W_{s(j)}}\{l o g \operatorname{det}(Q)\}, \\
& \text { s.t. }(16),(17),(18)
\end{aligned}
$$

With a small abuse of notation, denote $Q, W_{s(j)}$, and $L_{s(j)}=W_{s(j)} Q^{-1}$ as an optimal solution of (19). The obtained matrices $L_{s(j)}$ will be used in the next sections.

\section{Optimizing Prediction Dynamics with SATURATEd FeEdback}

\section{A. Augmented System with Saturated Feedback}

As written in previous sections, despite allowing efficient online computation [7], the time to regulate the plant to the origin using the control law (11) is much longer than necessary. To overcome this weakness, the following saturated control law is considered, $t=0,1,2, \ldots$

$$
u(k+t \mid k)=\operatorname{sat}(K x(k+t \mid k)+v(k+t \mid k))
$$

where $v(k+t \mid k) \in \mathbb{R}^{m}$ is an auxiliary variable that will be treated as a decision variable. Our problem is to select $v(k+t \mid k), t=0,1,2, \ldots$, at each time instant such that the resulting control law minimizes the cost (7), while at the same time, the feasible set of (10) is as large as possible.

Following the prediction dynamics method, assuming $v(k+t \mid k)$ is the output of an auxiliary dynamical system, i.e.,

$$
v(k+t \mid k)=H r(k+t \mid k)
$$

where $r(k+t \mid k) \in \mathbb{R}^{n_{r}}$ is the auxiliary state, $H \in \mathbb{R}^{m \times n_{r}}$ is variable to be calculated. The system matrix that links $r(k+t+1 \mid k)$ and $r(k+t \mid k)$ will be defined later.

For simplicity, the time index for $\theta$ is omitted. Substituting (20), (21) into (1), one obtains

$$
x(k+t+1 \mid k)=\sum_{i=1}^{q} \theta_{i}\left(A_{i} x(k+t \mid k)+B_{i} \operatorname{sat}\left(\left[\begin{array}{ll}
K & H
\end{array}\right] z(t+k \mid k)\right)\right)
$$

where $z(k+t \mid k)=\left[\begin{array}{ll}x(k+t \mid k)^{T} & r(k+t \mid k)^{T}\end{array}\right]^{T}$. Denote $H_{(j)}$ as the $j$ row of $H$. Using Lemma 2, one gets, $\forall i=\overline{1, q}$

$$
\left.\begin{array}{l}
B_{i} \operatorname{sat}\left\{\left[\begin{array}{cc}
K & H
\end{array}\right] z(k+t \mid k)\right) \in \\
\in \operatorname{Co}\left\{\left(\sum_{j \in S_{s}^{c}} B_{i(j)}\left[\begin{array}{ll}
K_{(j)} & H_{(j)}
\end{array}\right]+\sum_{j \in S_{s}} B_{i(j)}\left[\begin{array}{ll}
L_{s(j)} & D_{s(j)}
\end{array}\right]\right) z(k+t \mid k), s=\overline{1,2^{m}}\right.
\end{array}\right\}
$$


for all $z(k+t \mid k)$ such that, $\forall s=\overline{1,2^{m}}$

$$
-1 \leq\left[\begin{array}{ll}
L_{s(j)} & D_{s(j)}
\end{array}\right] z(k+t \mid k) \leq 1, \forall j=\overline{1, m}
$$

In (23), (24), $D_{s(j)} \in \mathbb{R}^{1 \times n_{r}}$ are unknown variables. The system matrix that links $r(k+t+1 \mid k)$ and $r(k+t \mid k)$ is defined as, $\forall t=0,1, \ldots$

$$
r(k+t+1 \mid k) \in C o\left\{\left(\sum_{i=1}^{q} \theta_{i} \Psi_{i s}\right) r(k+t \mid k), s=\overline{1,2^{m}}\right\}
$$

where $\Psi_{i s} \in \mathbb{R}^{n_{r} \times n_{r}}$ are variables to be calculated. Using (22), (23), (25), one gets, $\forall z(k+t \mid k)$ satisfying (24),

$$
\begin{aligned}
& z(k+t+1 \mid k) \in C o\left\{\left(\sum_{i=1}^{q} \theta_{i} \mathcal{A}_{i s}\right) z(k+t \mid k), \forall s=\overline{1,2^{m}}\right\}, \\
& \mathcal{A}_{i s}=\left[\begin{array}{cc}
\Omega_{i s} & \left(\sum_{j \in S_{s}^{c}} B_{i(j)} H_{(j)}+\sum_{j \in S_{s}} B_{i(j)} D_{s(j)}\right) \\
\mathbf{0}_{n_{r} \times n} & \Psi_{i s}
\end{array}\right] \\
& \Omega_{i s}=A_{i}+\sum_{j \in S_{s}^{c}} B_{i(j)} K_{(j)}+\sum_{j \in S_{s}} B_{i(j)} L_{s(j)}
\end{aligned}
$$

Remark 2: Note that the prediction dynamics (25) depends not only on the model uncertainties, but also on the saturated inputs. As will be shown in the numerical examples, this additional design freedom indeed leads to a considerable performance improvement.

\section{B. Domain of Attraction Characterization}

This section concerns the problem of characterizing the domain of attraction for the system (26) with the state constraints (3). The input constraints (2) are naturally satisfied because of the applied saturation function.

Following [11], define the matrices $\mathcal{P}, \mathcal{P}^{-1}$ partitioned into blocks as

$$
\mathcal{P}=\left[\begin{array}{cc}
X^{-1} & X^{-1} U \\
U^{T} X^{-1} & (?)
\end{array}\right], \mathcal{P}^{-1}=\left[\begin{array}{cc}
Y & V \\
V^{T} & (?)
\end{array}\right]
$$

where $X \in \mathbb{R}^{n \times n}, Y \in \mathbb{R}^{n \times n}$ are symmetric matrices, $U \in \mathbb{R}^{n \times n_{r}}, V \in \mathbb{R}^{n \times n_{r}}$, and (?) denote blocks in $\mathcal{P}, \mathcal{P}^{-1}$ that are uniquely determined by $X, Y, U, V$. Define also

$$
M_{(j)}=H_{(j)} V^{T}, N_{s(j)}=D_{s(j)} V^{T}, T_{i s}=U \Psi_{i s} V^{T}
$$

Note that $\mathcal{P} \mathcal{P}^{-1}=I$ implies

$$
U V^{T}=X-Y
$$


If $n_{r}<n$, then equation (29) imposes a nonconvex rank constraint, i.e., $\operatorname{rank}(X-Y)=n_{r}$. Hence only the case $n_{r} \geq n$ is considered.

The following theorem provides the main result of the paper. It establishes the theoretical support of the algorithm proposed to obtain an estimation of the domain of attraction for (24).

Theorem 1: Suppose that there exist symmetric matrices $X \in \mathbb{R}^{n \times n}, Y \in \mathbb{R}^{n \times n}$, matrices $M_{(j)} \in \mathbb{R}^{1 \times n}, N_{s(j)} \in \mathbb{R}^{1 \times n}, T_{i s} \in \mathbb{R}^{n \times n}$, such that the following LMIs hold, $\forall i=\overline{1, q}, \forall s=\overline{1,2^{m}}$

$$
\begin{aligned}
& {\left[\begin{array}{cc}
{\left[\begin{array}{cc}
Y & X \\
X & X
\end{array}\right]} & {\left[\begin{array}{cc}
\left(\Omega_{i s} Y+\Gamma_{i s}\right) & \Omega_{i s} X \\
\left(\Omega_{i s} Y+\Gamma_{i s}+T_{i s}\right) & \Omega_{i s} X
\end{array}\right]} \\
(*) & {\left[\begin{array}{cc}
Y & X \\
X & X
\end{array}\right]}
\end{array}\right] \succeq 0,}
\end{aligned}
$$

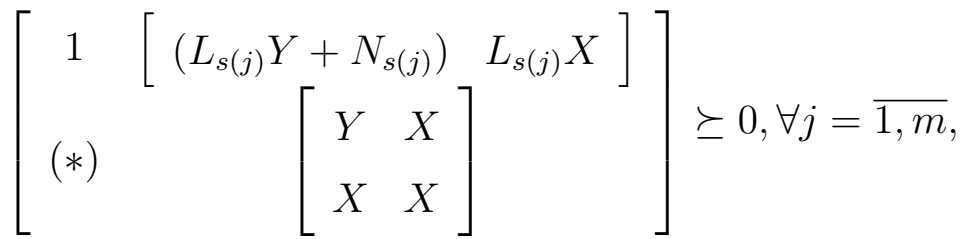

$$
\begin{aligned}
& {\left[\begin{array}{cc}
1 & {\left[\begin{array}{cc}
f_{l} Y & f_{l} X
\end{array}\right]} \\
(*) & {\left[\begin{array}{cc}
Y & X \\
X & X
\end{array}\right]}
\end{array}\right] \succeq 0, \forall l=\overline{1, n_{c}},}
\end{aligned}
$$

where $\Gamma_{i s}=\sum_{j \in S_{s}^{c}} B_{i(j)} M_{j}+\sum_{j \in S_{s}} B_{i(j)} N_{s(j)}$, then $\mathcal{E}(\mathcal{P})$ is robustly invariant for (24) and constraintadmissible with respect to (3).

Proof: Using Lemma 1 , one has $\mathcal{E}(\mathcal{P}) \subseteq \mathcal{L}(F)$ if and only if

$$
\left[\begin{array}{cc}
1 & {\left[\begin{array}{ll}
f_{l} & \mathbf{0}_{1 \times n_{r}}
\end{array}\right]} \\
(*) & \mathcal{P}
\end{array}\right] \succeq 0, \forall l=\overline{1, n_{c}}
$$

Pre- and post-multiplication of (33) by

$$
\left[\begin{array}{cc}
1 & \mathbf{0}_{1 \times\left(n+n_{r}\right)} \\
(*) & \mathcal{G}^{T}
\end{array}\right],\left[\begin{array}{cc}
1 & \mathbf{0}_{1 \times\left(n+n_{r}\right)} \\
(*) & \mathcal{G}
\end{array}\right]
$$

with $\mathcal{G}=\left[\begin{array}{cc}Y & X \\ V^{T} & \mathbf{0}_{n_{r} \times n}\end{array}\right]$ one obtains (32). Hence $\mathcal{E}(\mathcal{P})$ is admissible with respect to (3). Note that (26) holds if and only if (24) is satisfied. In other words, $\forall z \in \mathcal{E}(\mathcal{P})$

$$
-1 \leq\left[\begin{array}{ll}
L_{s(j)} & D_{s(j)}
\end{array}\right] z \leq 1
$$


Using Lemma 1, this condition is equivalent to

$$
\left[\begin{array}{cc}
1 & {\left[\begin{array}{cc}
L_{s(j)} & D_{s(j)}
\end{array}\right]} \\
(*) & \mathcal{P}
\end{array}\right] \succeq 0
$$

Thus, by pre- and post- multiplying with

$$
\left[\begin{array}{cc}
1 & \mathbf{0}_{1 \times\left(n+n_{r}\right)} \\
(*) & \mathcal{G}^{T}
\end{array}\right],\left[\begin{array}{cc}
1 & \mathbf{0}_{1 \times\left(n+n_{r}\right)} \\
(*) & \mathcal{G}
\end{array}\right]
$$

one gets (31). Therefore, equation (26) holds under (31). For invariance of $\mathcal{E}(\mathcal{P})$, it is required that

$$
z(k+1 \mid k)^{T} \mathcal{P} z(k+1 \mid k) \leq z(k \mid k)^{T} \mathcal{P} z(k \mid k)
$$

Thus, using (26), one gets $\mathcal{A}_{i s}^{T} \mathcal{P} \mathcal{A}_{i s} \preceq \mathcal{P}$. Or equivalently, with Schur complement,

$$
\left[\begin{array}{cc}
\mathcal{P} & \mathcal{A}_{i s}^{T} \mathcal{P} \\
\mathcal{P} \mathcal{A}_{i s} & \mathcal{P}
\end{array}\right] \succeq 0
$$

Pre- and and post-multiplication of (34) by

$$
\left[\begin{array}{cc}
\mathcal{G}^{T} & \mathbf{0}_{2 n \times\left(n+n_{r}\right)} \\
(*) & \mathcal{G}^{T}
\end{array}\right],\left[\begin{array}{cc}
\mathcal{G} & \mathbf{0}_{\left(n+n_{r}\right) \times 2 n} \\
(*) & \mathcal{G}
\end{array}\right]
$$

one gets

$$
\left[\begin{array}{cc}
\mathcal{G}^{T} \mathcal{P} \mathcal{G} & \mathcal{G}^{T} \mathcal{A}_{i s}^{T} \mathcal{P} \mathcal{G} \\
\mathcal{G}^{T} \mathcal{P} \mathcal{A}_{i s} \mathcal{G} & \mathcal{G}^{T} \mathcal{P} \mathcal{G}
\end{array}\right] \succeq 0
$$

thus, using (28), one gets (30). The proof is complete.

The projection of $\mathcal{E}(\mathcal{P})$ onto the $x$-subspace is given by $\mathcal{E}\left(Y^{-1}\right)=\left\{x \in \mathbb{R}^{n}: x^{T} Y^{-1} x \leq 1\right\}$, see Fig. 1. Note that the optimal value of $Y$ is independent of $n_{r}$ if $n_{r}>n$. Therefore $n_{r}=n$ is assumed in the rest of the paper. With $n_{r}=n$ and for given $M_{(j)}, N_{s(j)}$ and $T_{i s}$, the solution to $(28)$ is

$$
H_{(j)}=M_{(j))} V^{-T}, D_{s(j)}=N_{s(j)} V^{-T}, \Psi_{i s}=U^{-1} T_{i s} V^{-T}
$$

For any given saturated stabilizing control law $u(k)=\operatorname{sat}(\tilde{K} x(k))$, define $\mathcal{E}(\tilde{S})$ as the maximal invariant ellipsoid for the system

$$
x(k+1)=A(k) x(k)+B(k) \operatorname{sat}(\tilde{K} x(k))
$$

and constraint-admissible with respect to the constraints (3). The following theorem holds 


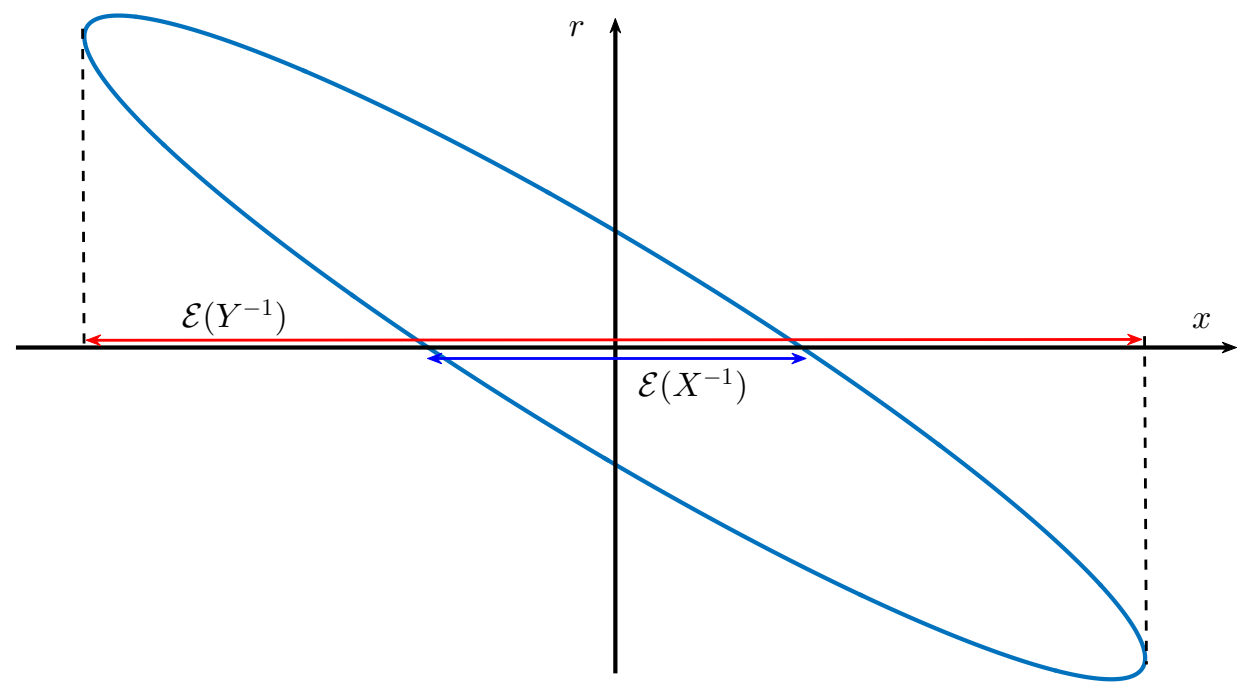

Fig. 1: Graphical illustrations for the projection $\mathcal{E}\left(Y^{-1}\right)$ (solid red) of $\mathcal{E}(\mathcal{P})$ onto the $x$ state space and for the cut $\mathcal{E}\left(X^{-1}\right)$ (solid blue) of $\mathcal{E}(\mathcal{P})$ though $r=0$ in 2D.

Theorem 2: Among all solutions that satisfy (30), (31), (32), the set $\mathcal{E}(\mathcal{P})$ can be optimized in such a way that $\mathcal{E}(\tilde{S}) \subseteq \mathcal{E}\left(Y^{-1}\right)$.

Proof: By choosing $H=\tilde{K}-K$, the control law (20) becomes

$$
u(k)=\operatorname{sat}(K x(k)+(\tilde{K}-K) r(k))
$$

Note that $r(k)$ is a decision variable, that we can set to any value as far as the constraints on $u(k)$ and $z(k)$ are satisfied. Due the applied saturation function, the constraints on $u(k)$ are automatically fulfilled. The constraints on $z(k)$ are

$$
\left|\left[\begin{array}{ll}
f_{l} & \mathbf{0}_{1 \times n}
\end{array}\right]\left[\begin{array}{c}
x(k) \\
r(k)
\end{array}\right]\right| \leq 1, \forall l=1,2, \ldots, n_{c}
$$

or equivalently, $\left|f_{l} x(k)\right| \leq 1$. Hence, one can consider $r(k)=x(k)$. In this case, one gets $u(k)=\operatorname{sat}(\tilde{K} x(k))$. The closed loop system under (20) is

$$
x(k+1)=A(k) x(k)+B(k) \operatorname{sat}(\tilde{K} x(k))
$$

It follows that $\mathcal{E}(\tilde{S}) \subseteq \mathcal{E}\left(Y^{-1}\right)$ as long as $\mathcal{E}(\tilde{S})$ is the maximal invariant set for (36) and constraint-admissible to (3). The proof is complete. 
Theorem 2 states that $\mathcal{P}$ can be optimized such that the maximal invariant set under any saturated feedback law is a subset of $\mathcal{E}\left(Y^{-1}\right)$.

\section{THE PROPOSED CONTROL METHOD}

In this section, we will first show how to calculate an upper bound of the cost $J(k)$, then we will introduce the new control scheme. Consider the following quadratic function

$$
V(z)=z^{T} \Phi z
$$

where $\Phi \in \mathbb{R}^{2 n \times 2 n}$ is chosen to satisfy, $\forall t=0,1, \ldots$

$$
\begin{aligned}
& V(z(k+t \mid k))-V(z(k+t+1 \mid k)) \geq \\
& \geq x(k+t \mid k)^{T} Q x(k+t \mid k)+u(k+t \mid k)^{T} R u(k+t \mid k)
\end{aligned}
$$

with $Q, R$ given in (7). Theorem 3 below will provide conditions to compute the matrix $\Phi$. For the moment, let us assume that $\Phi$ is known.

Since (26) is robustly asymptotically stable $\forall z \in \mathcal{E}(\mathcal{P})$, it follows that $z(\infty \mid k)=0$, and therefore $V(\infty \mid k)=0$. Summing (38) from $t=0$ to $t=\infty$, one obtains

$$
\begin{aligned}
& V(z(k \mid k)) \geq \\
& \sum_{t=0}^{\infty}\left(x(k+t \mid k)^{T} Q x(k+t \mid k)+u(k+t \mid k)^{T} R u(k+t \mid k)\right)
\end{aligned}
$$

The right hand side of (39) is the cost function $J(k)$ in (7). Hence $J(k) \leq V(z(k \mid k))$. In other words, $V(z(k \mid k))$ provides an upper bound of $J(k)$. Define

$$
\begin{aligned}
& \mathcal{K}_{(j)}=\left[\begin{array}{cc}
K_{(j)} & H_{(j)}
\end{array}\right], \mathcal{D}_{s(j)}=\left[\begin{array}{ll}
L_{s(j)} & D_{s(j)}
\end{array}\right] \\
& \Delta_{s}=\sum_{j \in S_{s}^{c}} e_{j} \mathcal{K}_{(j)}+\sum_{j \in S_{s}^{c}} e_{j} \mathcal{D}_{s(j)}
\end{aligned}
$$

Using (26), one obtains the following theorem concerns the existence of $\Phi$ that satisfies (38).

Theorem 3: There exists $\Phi$ satisfying (38) if and only if the following LMIs in $\Phi$ are feasible, $\forall i=\overline{1, q}, \forall s=\overline{1,2^{m}}$

$$
\mathcal{A}_{i s}^{T} \Phi \mathcal{A}_{i s}-\Phi+Q+\Delta_{s}^{T} R \Delta_{s} \preceq 0
$$

Using the Cauchy-Schwarz inequality, it is clear that there exists $\Phi \in \mathbb{R}^{2 n \times 2 n}$ that satisfies (41), if and only if there exists $\Phi$ in the following diagonal form

$$
\Phi=\left[\begin{array}{cc}
\Phi_{x x} & \mathbf{0}_{n \times n} \\
\mathbf{0}_{n \times n} & \Phi_{r r}
\end{array}\right]
$$


where $\Phi_{x x}, \Phi_{r r} \in \mathbb{R}^{n \times n}$ are symmetric matrices. Note that $\Phi$ is used to provide an upper bound of $J(k)$. The optimal $\Phi$ can be calculated by solving the following SDP problem

$$
\min _{\Phi_{x x}, \Phi_{r r}}\left\{\operatorname{trace}\left(\Phi_{x x}\right)+\operatorname{trace}\left(\Phi_{r r}\right)\right\} \text {, s.t. }(41)
$$

Let $\Phi_{x x}^{*}, \Phi_{r r}^{*}$ be the solution of (43). Consider the following optimization problem, at time $k$,

$$
\begin{aligned}
& \min _{r(k)}\left\{r(k)^{T} \Phi_{r r}^{*} r(k)\right\}, \\
& \text { s.t. }\left[x(k)^{T} \quad r(k)^{T}\right] \mathcal{P}\left[\begin{array}{l}
x(k) \\
r(k)
\end{array}\right] \leq 1
\end{aligned}
$$

Let $r^{*}(k)$ be the solution of (44). The control signal applied to (1) at time $k$ is

$$
u(k)=\operatorname{sat}\left(K x(k)+H r^{*}(k)\right)
$$

Theorem 4: Assume feasibility at the initial state, the control law (45) guarantees recursive feasibility and robust asymptotic stability.

Recursive feasibility proof: Since $\mathcal{E}\left(Y^{-1}\right)$ is the projection of $\mathcal{E}(\mathcal{P})$ onto the state space axis, it follows that $\exists r(k)$ such that $z(k)=\left[x(k)^{T} r(k)^{T}\right]^{T} \in \mathcal{E}(\mathcal{P}), \forall x(k) \in \mathcal{E}\left(Y^{-1}\right)$. Hence problem (44) is feasible at time $k$. Note that $\mathcal{E}(\mathcal{P})$ is robustly invariant for (1), and constraint-admissible to (3) under the control law (20). Hence $z(k+1)=\left[x(k+1)^{T} r(k+1)^{T}\right]^{T} \in \mathcal{E}(\mathcal{P})$. Therefore $x(k+1) \in \mathcal{E}\left(Y^{-1}\right)$. In other words, recursive feasibility is guaranteed.

Robust asymptotic stability proof: Consider the following Lyapunov function candidate

$$
\left.V^{*}(z(k))\right)=x(k)^{T} \Phi_{x x}^{*} x(k)+r^{*}(k)^{T} \Phi_{r r}^{*} r^{*}(k)
$$

Note that $V^{*}(k)=z^{*}(k)^{T} \Phi^{*} z^{*}(k)$. Applying (45) to the system (1), one obtains $z(k+1)$. Using (38), one gets

$$
V(z(k+1))-V^{*}(z(k)) \leq-x(k)^{T} Q x(k)-u(k)^{T} R u(k)
$$

Optimizing at time $k+1$ yields $r^{*}(k+1)^{T} \Phi_{r r}^{*} r^{*}(k+1) \leq r(k+1)^{T} \Phi_{r r}^{*} r(k+1)$. Hence $V^{*}(z(k+$ $1)) \leq V(z(k+1))$. It follows that

$$
V^{*}(z(k+1))-V^{*}(z(k)) \leq-x(k)^{T} Q x(k)-u(k)^{T} R u(k)
$$

Hence $V^{*}(z(k))$ is a Lyapunov function of the closed loop system with the control law (45). In other words, robust asymptotic stability is guaranteed. 
Remark 3: Using the proof of Theorem 4, it is clear that the control law (45) guarantees robust asymptotic stability for any $\Phi_{r r}$ that satisfies, $\forall i=\overline{1, q}, \forall s=\overline{1,2^{m}}$

$$
\Phi_{r r}-\Psi_{i s}^{T} \Phi_{r r} \Psi_{i s} \prec 0
$$

Decompose $\mathcal{P}$ as

$$
\mathcal{P}=\left[\begin{array}{ll}
P_{x x} & P_{x r} \\
P_{x r}^{T} & P_{r r}
\end{array}\right]
$$

where $P_{x x}=X^{-1}, P_{x r}=X^{-1} U$. The matrix $P_{r r}$ is obtained by using $\mathcal{P P}^{-1}=I_{2 n}$. Hence $P_{r r}=-U^{T} X^{-1} Y V^{-T}$. The cut of $\mathcal{E}(\mathcal{P})$ through $r=0$ is given as $\mathcal{E}\left(X^{-1}\right)=\left\{x \in \mathbb{R}^{n}\right.$ : $\left.x^{T} X^{-1} x \leq 1\right\}$, see Fig. 1. Rewrite (44) as

$$
\begin{aligned}
& \min _{r(k)}\left\{r(k)^{T} \Phi_{r r}^{*} r(k)\right\}, \\
& \text { s.t. } r(k)^{T} P_{r r} r(k)+2 r(k)^{T} P_{x r}^{T} x(k)+x(k)^{T} P_{x x} x(k) \leq 1
\end{aligned}
$$

Clearly, (49) is a convex optimization problem, for which the solution is unique. As noticed in [8], problem (49) has a very nice geometrical interpretation. Indeed the solution to (49) defines the shortest distance of an ellipsoid from the origin for a given $x(k)$. To see this, define $\mathcal{E}_{r}$ as a feasible set, to which $r(k)$ must belong. Clearly, $\mathcal{E}_{r}$ is an ellipsoid. If $x(k) \in \mathcal{E}\left(P_{x x}\right)=\{x \in$ $\left.\mathbb{R}^{n}: x^{T} P_{x x} x \leq 1\right\}$, then $\mathcal{E}_{r}$ contains the origin. Hence (49) has a trivial solution $r^{*}(k)=0$. In this case, (45) becomes $u(k)=\operatorname{sat}(K x(k))$, i.e., the robust optimal saturated LQ controller. Otherwise, if $\mathcal{E}_{r}$ does not contain the origin, then $r^{*}(k)$ must lie on the border of $\mathcal{E}_{r}$ that is closest to the origin in the $\Phi_{r r}^{*}$-norm sense. The solution of (49) can be found by using a univariate Newton-Raphson procedure with quadratic convergence rate via a Lagrange multiplier [8].

$\mathcal{E}\left(X^{-1}\right)$ should be maximized in the interest of the closed-loop performance, because this is the region on which $u(k)=\operatorname{sat}(K x(k))$ is feasible. On the other hand, $\mathcal{E}\left(Y^{-1}\right)$ should also be maximized, since recursive feasibility and robust asymptotic stability of the control law (45) are guaranteed for $x(k) \in \mathcal{E}\left(Y^{-1}\right)$. To address both objectives, the following SDP problem is used

$$
\begin{aligned}
& \left.\left.\max _{X, Y, M_{(j)}, N_{s(j)}, T_{i s}}(\operatorname{logdet}(Y))+\alpha \operatorname{cogdet}(X)\right)\right) \\
& \text { s.t. }(30),(31),(32)
\end{aligned}
$$

for some tuning constant $\alpha \geq 0$.

In summary, the proposed control policy consists of two stages: offline stage and online stage.

Remark 4: Even though the optimization problems (19), and (43), and (50) are convex, they are NP-hard [16]. The result is that for system (1) with parametric uncertainty and with hundreds of states, solving these problems can become computationally prohibitive. 
OFFLINE:

1: $\quad$ Select the matrix gain $K$

2 : $\quad$ Obtain matrices $L_{s(j)}$ by solving (19)

3: $\quad$ Obtain matrices $\mathcal{P}, \Psi_{i s}, H$ by solving (50)

4: $\quad$ Obtain $\Phi_{r r}$ by solving (43)

\section{ONLINE: At each time instant $k \geq 0$}

1: $\quad$ Measure or estimate the state $x(k)$

2: Obtain $r^{*}(k)$ by solving (49)

3: $\quad$ Apply $u(k)=\operatorname{sat}\left(K x(k)+H r^{*}(k)\right)$ to (1)

Theorem 5: The control law (45) can be represented as a continuous and saturated function of the state.

Proof: Using the Langrange multiplier method, it follows that the solution of (49) satisfies $\Phi_{r r}^{*} r^{*}(k)+\lambda\left(Q_{r r} r^{*}(k)+Q_{x r}^{T} x(k)\right)=0$, where $\lambda \geq 0$ is a Langrange multiplier. Therefore

$$
r^{*}(k)=-\lambda\left(\Phi_{r r}^{*}+\lambda Q_{r r}\right)^{-1} Q_{x r}^{T} x(k)
$$

Substituting (51) into (45), one gets

$$
u(k)=\operatorname{sat}\left(\left(K-\lambda(H-K)\left(\Phi_{r r}^{*}+\lambda Q_{r r}\right)^{-1} Q_{x r}^{T}\right) x(k)\right)
$$

Hence the control law (45) is a continuous and saturated function of state.

\section{EXAMPLES}

This section demonstrates the potential benefit of the new control law by simulations of two example systems. The CVX toolbox [17] was used to solve the SDP optimization problems.

\section{A. Example 1}

This example is taken from [7]. Consider (1) with $A=\left[\begin{array}{rr}1 & 0.1 \\ 0 & 1\end{array}\right], B=\left[\begin{array}{r}0 \\ 0.0787\end{array}\right]$. The constraints on $x(k)$ are $-10 \leq x_{1} \leq 10,-3 \leq x_{2} \leq 3$. The weighting matrices are $Q=\operatorname{diag}\left(\left[\begin{array}{ll}1 & 0\end{array}\right]\right), R=0.01$. The size of $\mathcal{E}\left(Y^{-1}\right), \mathcal{E}\left(X^{-1}\right)$ for different $\alpha$ are obtained by solving (50), and given in Table I. For comparison, Table I also shows $\mathcal{E}\left(Y^{-1}\right), \mathcal{E}\left(X^{-1}\right)$ using [11]. We can see that the proposed approach significantly reduces the design conservativeness, resulting 
TABLE I: Invariant set size for different $\alpha$ for example 1.

\begin{tabular}{|c|c|c|c|c|c|c|}
\hline$\alpha$ & 0.01 & 0.1 & 1 & 10 & 100 & 1000 \\
\hline $\log \operatorname{det}(X)$ using $(50)$ & -3.19 & -1.88 & -1.05 & -0.87 & -0.86 & -0.86 \\
\hline $\log \operatorname{det}(X)$ in $[11]$ & -8.37 & -7.07 & -6.02 & -5.69 & -5.67 & -5.67 \\
\hline $\log \operatorname{det}(Y)$ using $(50)$ & 6.79 & 6.75 & 6.42 & 6.02 & 5.95 & 5.94 \\
\hline $\log \operatorname{det}(Y)$ in $[11]$ & 6.79 & 6.74 & 6.31 & 5.48 & 5.13 & 5.08 \\
\hline
\end{tabular}

in a much larger feasible sets. Choosing $\alpha=1$, Fig. 2 shows the feasible sets for our approach (solid blue), for [11] (dash-dot green), and for the standard dual mode MPC (dashed red). The MPC prediction horizon is $N_{p}=35$. For the initial condition $x(0)=\left[\begin{array}{ll}-2 & 2\end{array}\right]^{T}$, Fig. 3 presents

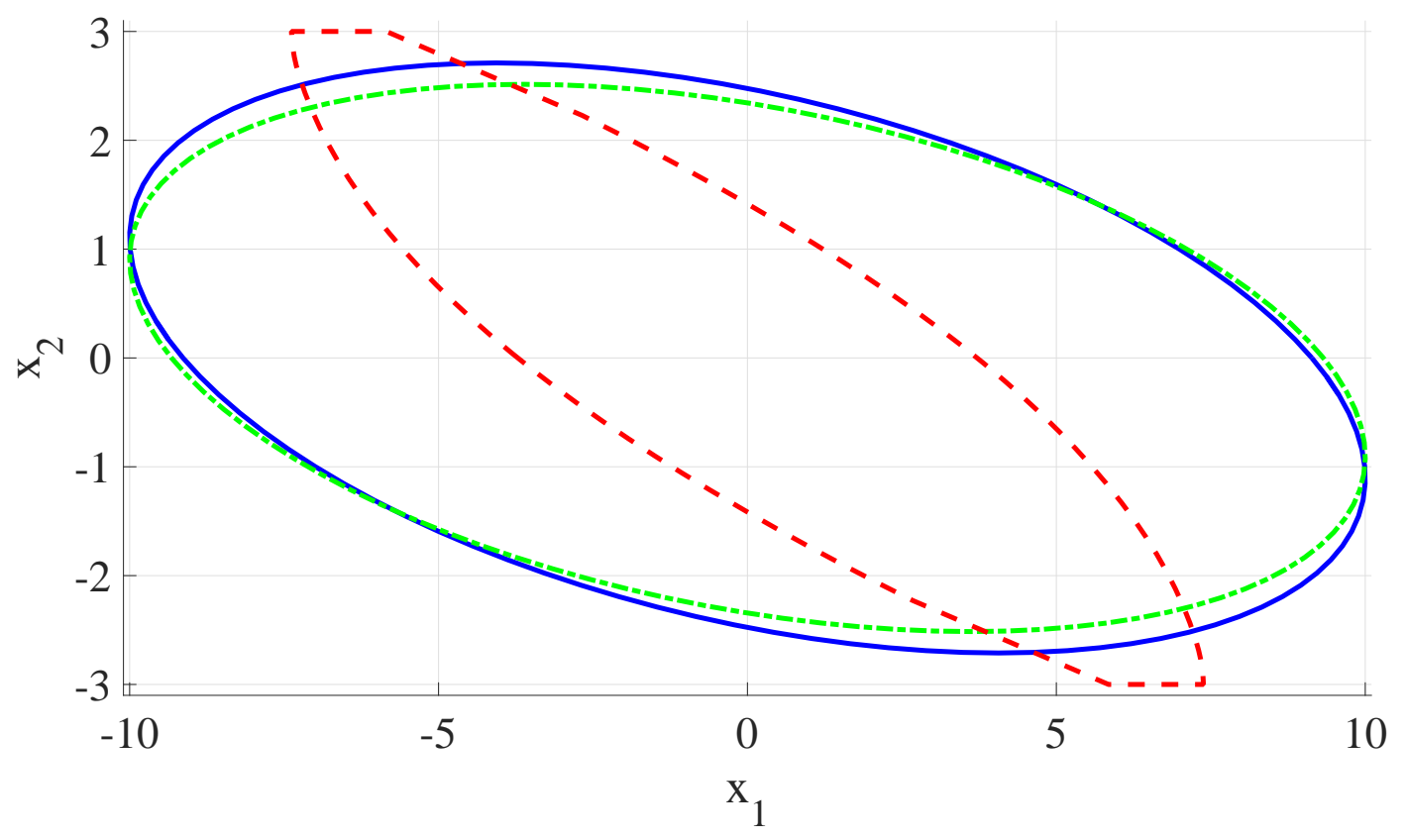

Fig. 2: Feasible set for our approach (solid blue), for [11] (dash-dot green), and for MPC (dashed red) for example 1.

the state and input trajectories of the closed loop system as functions of time using our approach (solid blue), [11] (dash-dot green), and the MPC (dashed red). Note that the performances of our controller and of MPC are almost identical.

Using the TIC / TOC function of Matlab 2015b, we found that the on-line computation times 

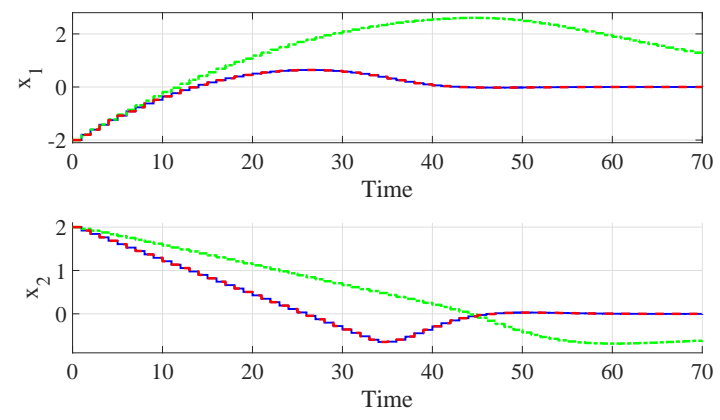

(a) State

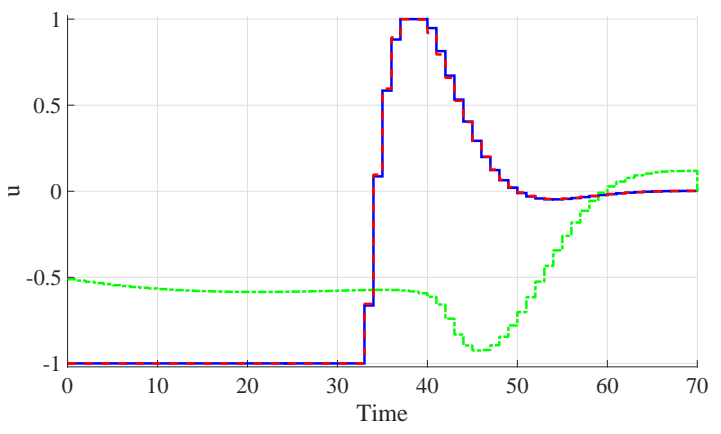

(b) Input

Fig. 3: State and input trajectories for our approach (solid blue), for [11] (dash-dot green), and for MPC (dashed red) for example 1.

for one discretization interval were $4.1424 \times 10^{-4}[s], 3.7214 \times 10^{-4}[s]$, and $0.0110[s]$ for our approach, for [11], and for MPC, respectively.

\section{B. Example 2}

This example is concerned with the fourth-order two mass-spring system taken from Problem 4 of the benchmark problems for robust control design in [18]. Using Euler's first order approximation for the derivative with a sampling time of $0.1 s$, the discrete-time system (1) is obtained with

$$
A(k)=\left[\begin{array}{rrrr}
1.0 & 0 & 0.1 & 0 \\
0 & 1.0 & 0 & 0.1 \\
-0.1 \frac{\mathbf{K}(k)}{m_{1}} & 0.1 \frac{\mathbf{K}(k)}{m_{1}} & 1.0 & 0 \\
0.1 \frac{\mathbf{K}(k)}{m_{2}} & -0.1 \frac{\mathbf{K}(k)}{m_{2}} & 0 & 1.0
\end{array}\right], B=\left[\begin{array}{r}
0 \\
0 \\
\frac{0.1}{m_{1}} \\
0
\end{array}\right]
$$

Here $m_{1}=m_{2}=1$ are the masses of the two bodies. $\mathbf{K}(k)$ is the uncertain spring coefficient, $\mathbf{K}(k)=\theta(k) K_{1}+(1-\theta(k)) K_{2}$ where $K_{1}=1, K_{2}=3$, and $\theta(k) \in[0,1]$ is a uniformly distributed pseudo-random number. The constraints are $-5 \leq x_{1} \leq 5$. The weighting matrices are $Q=\operatorname{diag}\left(\left[\begin{array}{llll}1 & 0 & 0 & 0\end{array}\right]\right), R=0.09$. The size of the ellipsoids $\mathcal{E}\left(Y^{-1}\right), \mathcal{E}\left(X^{-1}\right)$ for different $\alpha$ are given in Table II. This table also shows the size of $\mathcal{E}\left(Y^{-1}\right), \mathcal{E}\left(X^{-1}\right)$ using [11]. For the initial condition $x(0)=\left[\begin{array}{llll}-2.4149 & -2.4335 & -0.0534 & 0.0903\end{array}\right]^{T}$, Fig. 4 presents the state and input trajectories of the closed loop system as functions of time using our approach (solid blue), using [2] (dashed red), and using [11] (dash-dot green) with $\alpha=1$. Fig. 4 also presents the 
TABLE II: Invariant set size for different $\alpha$ for example 2 .

\begin{tabular}{|c|c|c|c|c|c|}
\hline$\alpha$ & 0.01 & 0.1 & 1 & 10 & 100 \\
\hline $\log \operatorname{det}(X)$ using (50) & -18.48 & -10.00 & -7.47 & -7.38 & -7.38 \\
\hline $\log \operatorname{det}(X)$ in [11] & -23.61 & -15.07 & -10.89 & -10.63 & -10.62 \\
\hline $\log \operatorname{det}(Y)$ using (50) & -0.33 & -0.65 & -1.13 & -1.37 & -1.45 \\
\hline $\log \operatorname{det}(Y)$ in [11] & -0.33 & -0.65 & -1.77 & -2.34 & -2.49 \\
\hline
\end{tabular}
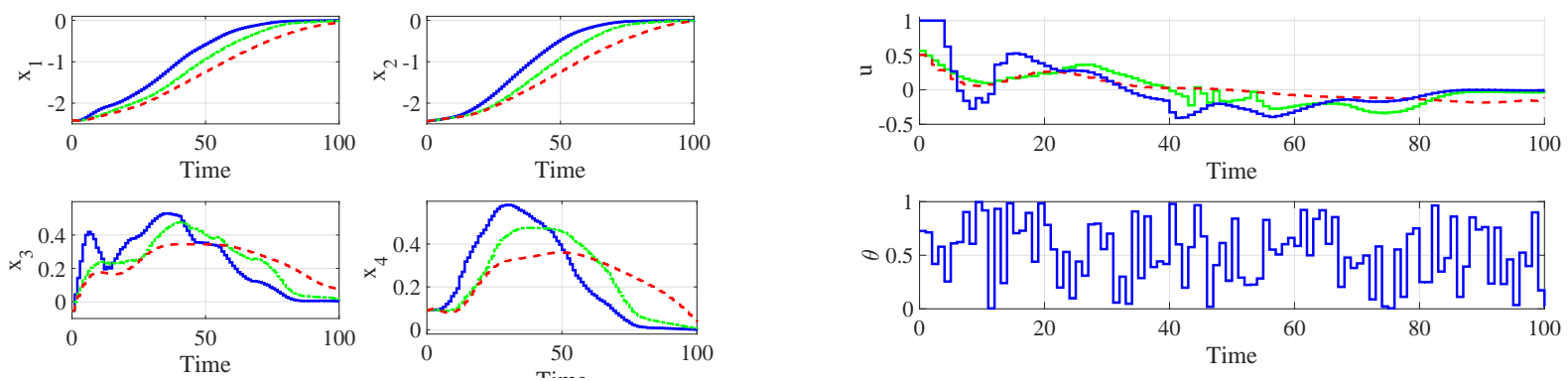

(a) State

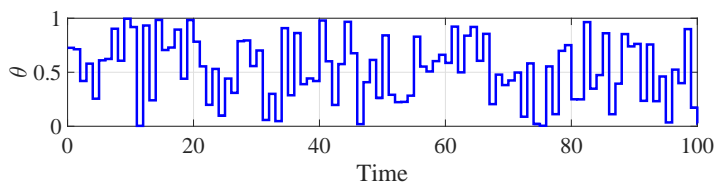

(b) Input and $\theta$ realization

Fig. 4: State trajectories, input trajectories, and $\theta$ realization for our approach (solid blue), for [2] (dashed red), and for [11] (dash-dot green) for example 2.

realization of $\theta(k)$. Note that a solution of a SDP problem is required at each time instant for [2].

Finally, using the TIC / TOC function, the on-line computation times for one discretization interval were $2.9275 \times 10^{-4}[s], 0.3363[s]$, and $4.9217 \times 10^{-4}[s]$ for our approach, for [2], and for [11], respectively.

\section{CONCLUSION}

A new predictive control method is introduced for discrete-time uncertain and/or time-varying linear systems with state and input constraints. To fully utilize the capacity of actuators, a saturated nonlinear feedback policy is used. The prediction dynamics is allowed to depend not only on the model uncertainties but also on the saturated inputs. It is shown that the feasible invariant set for the new approach can be equal to the maximal invariant ellipsoid under any saturated control law. A continuous and saturated piecewise linear control law is provided which 
not only guarantees recursive feasibility and robust asymptotic stability, but is also optimal for the state near the origin. The implementation of the new control law requires a minimal amount of computation, yet, for the two simulation examples, delivered optimal performance.

\section{REFERENCES}

[1] D. Q. Mayne, "Model predictive control: Recent developments and future promise," Automatica, vol. 50, no. 12, pp. 2967-2986, 2014.

[2] M. V. Kothare, V. Balakrishnan, and M. Morari, "Robust constrained model predictive control using linear matrix inequalities," Automatica, vol. 32, no. 10, pp. 1361-1379, 1996.

[3] J. H. Lee and Z. Yu, "Worst-case formulations of model predictive control for systems with bounded parameters," Automatica, vol. 33, no. 5, pp. 763-781, 1997.

[4] A. Bemporad, F. Borrelli, and M. Morari, "Min-max control of constrained uncertain discrete-time linear systems," IEEE Transactions on Automatic Control, vol. 48, no. 9, pp. 1600-1606, 2003.

[5] D. Angeli, A. Casavola, G. Franzè, and E. Mosca, "An ellipsoidal off-line MPC scheme for uncertain polytopic discrete-time systems," Automatica, vol. 44, no. 12, pp. 3113-3119, 2008.

[6] L. Imsland, J. A. Rossiter, B. Pluymers, and J. Suykens, "Robust triple mode MPC," International Journal of Control, vol. 81, no. 4, pp. 679-689, 2008.

[7] B. Kouvaritakis, M. Cannon, and J. A. Rossiter, "Who needs QP for linear MPC anyway?” Automatica, vol. 38, no. 5, pp. 879-884, 2002.

[8] B. Kouvaritakis, J. A. Rossiter, and J. Schuurmans, "Efficient robust predictive control," IEEE Transactions on Automatic Control, vol. 45, no. 8, pp. 1545-1549, 2000.

[9] S. Drageset, L. Imsland, and B. A. Foss, "Efficient model predictive control with prediction dynamics," in European Control Conference. IEEE, 2003, pp. 1087-1092.

[10] L. Imsland, N. Bar, and B. A. Foss, "More efficient predictive control," Automatica, vol. 41, no. 8, pp. 1395-1403, 2005.

[11] M. Cannon and B. Kouvaritakis, "Optimizing prediction dynamics for robust MPC," IEEE Transactions on Automatic Control, vol. 50, no. 11, pp. 1892-1897, 2005.

[12] S. Tarbouriech, G. Garcia, J. M. G. da Silva Jr, and I. Queinnec, Stability and stabilization of linear systems with saturating actuators. Springer Science \& Business Media, 2011.

[13] G. E. Dullerud and F. Paganini, A course in robust control theory: a convex approach. Springer Science \& Business Media, 2013, vol. 36.

[14] T. Hu, Z. Lin, and B. M. Chen, "Analysis and design for discrete-time linear systems subject to actuator saturation," Systems \& Control Letters, vol. 45, no. 2, pp. 97-112, 2002.

[15] T. Alamo, A. Cepeda, and D. Limon, "Improved computation of ellipsoidal invariant sets for saturated control systems," in 44th IEEE Conference on Decision and Control, and European Control Conference. IEEE, 2005, pp. 6216-6221.

[16] V. D. Blondel and J. N. Tsitsiklis, "A survey of computational complexity results in systems and control," Automatica, vol. 36, no. 9, pp. 1249-1274, 2000.

[17] M. Grant and S. Boyd, "CVX: Matlab software for disciplined convex programming, version 2.1," 2014.

[18] B. Wie and D. S. Bernstein, "Benchmark problems for robust control design," Journal of Guidance, Control, and Dynamics, vol. 15 , no. 5, pp. 1057-1059, 1992. 